\section{Microplane Model for Progressive Fracture of CONCRETE AND ROCK}

By Zdeněk P. Bažant,, ${ }^{1}$ F. ASCE and Byung H. Oh, ${ }^{2}$ A. M. ASCE

ABstaAct: A constitutive model for a brittle aggregate material that undergoes progressive tensile fracturing or damage is presented. It is assumed that the normal stress on a plane of any orientation within the material, called the microplane, is a function of only the normal strain on the same microplane. This strain is further assumed to be equal to the resolved component of the macroscopic strain tensor, while the stress on the microplane is not equal to the resolved component of the macroscopic stress tensor. The normal strain on a microplane may be interpreted as the sum of the elastic strain and of the opening widths (per unit length) of all microcracks of the same orientation as the microplane. An additional volumetric elastic strain is introduced to adjust the elastic Poisson ratio to a desired value. An explicit formula which expresses the langent stifness of the material as an integral over the surface of a unit hemisphere is derived from the principle of virtual work. The model can represent experimentally observed unaxial tensile strain-softening behavior, and the stress reduces to zero as the strain becomes sufficiently large. Due to various combinations of loading and unloading on individual microplanes, the response of the model is path-dependent. Since the tensorial invariance restrictions are always satisfied by the microplane system, the model can be applied to progressive fracturing under rotating principal stress directions. This type of application is the main purpose of the model.

\section{INTRODUCTION}

Tensile strain softening, i.e., a decrease of stress at increasing strain, is a characteristic property of concretes and rocks. This phenomenon, observable in a stable state only if the specimen size is not too large and if the testing machine is much stiffer than the specimen, is particularly important for fracture and must be taken into account if the deviations from linear elastic fracture mechanics predictions should be described realistically

A simple triaxial strain-softening formulation has been shown capable of modeling the existing test data on the fracture of concrete and rock $(4,8)$. This formulation, however, is applicable only in those situations for which the principal stress directions do not rotate during the strainsoftening process. Such a situation may be assumed for many practical problems, but not for some important ones. For example, if first vertical normal stress causes only a partial cracking (strain softening), and the fracture is subsequently completed when a horizontal shear stress is superimposed (which may happen when a shear wave follows a tensile wave), it is necessary to describe strain-softening triaxial behavior under the conditions of a rotating principal stress direction. This poses diffi-

'Prof. of Civ. Engrg. and Dir., Center for Concrete and Geomaterials, The Technological Inst., Northwestern Univ., Evanston, Ill. 60201

${ }^{2}$ Postdoctoral Assoc., Northwestern Univ.; presently Asst. Prof. of Civ. Engrg., Seoul National Univ., Seoul, Korea.

Note.-Discussion open until September 1, 1985. To extend the closing date one month, a written request must be filed with the ASCE Manager of Journals. The manuscript for this paper was submitted for review and possible publication on February 13, 1983. This paper is part of the Journal of Engineering Mechanics, Vol. 111, No. 4, April, 1985. CASCE, ISSN 0733-9399/85/0004-0559/\$01.00. Paper No. 19642 culties in regard to tensorial invariance restrictions. The purpose of this paper is to present one possible model that satisfies the invariance restrictions and also realistically describes measured strain-softening curves.

The invariance requirements, such as those of isotropy, are usually met in constitutive modeling of nonlinear solids either by considering appropriate tensor polynomials or by using scalar loading functions and inelastic potentials that depend only on the invariants of the stress tensor. While these classical approaches should, in theory, work, they run into practical difficulties in some situations. The tensile strain softening is one of them.

Another, more powerful possibility is to specify the constitutive properties by a relation between the stresses and strains acting on a plane of arbitrary orientation in the material. The appropriate tensorial invariance restrictions can then be satisfied by subsequently considering a suitable distribution of orientations of these planes within the material For example, to obtain isotropy, each orientation must be equally frequent.

The idea of defining the inelastic behavior independently on planes of different orientations within the material, and then in some way superimposing the contributions from all the planes, has a long history. Building on the ideas of Taylor (23), Batdorf and Budiansky (3) formulated the slip theory of plasticity, in which the stresses acting on various slip planes are assumed to be the resolved components of the macroscopic applied stress tensor, and the plastic strains (slips) from all slip planes are superimposed. The same superposition of inelastic strains was used in the so-called multilaminate models of Zienkiewicz, et al. (26), and Pande, et al. $(17,18,24)$. Various other works (12) used a similar approach. It seems, however, that no model of this type exists which could describe tensile strain softening. Development of such a model, first proposed in general terms in Ref. 5 , is the objective of the present work, based on a 1983 report (10) briefly summarized at a recent conference (9). An extension, in which an application of the present approach to crack shear is developed, has already been published in Ref. 6 (even though it was submitted for publication nine months later).

\section{Basic HYPotheses}

In a manner similar to the slip theory of plasticity, we characterized the constitutive properties of the material by an independent relation between the stresses and strains acting on a plane of any orientation within the material, and assume that this relation is the same for the planes of all orientations. However, for the interaction belween the planes of various orientations, we must adopt rules which differ from those used in the slip theory in order to be able to model strain softening. By contrast with the slip theory, we cannot assume that the stress components on each of the aforementioned planes are equal to the resolved component of the macroscopic stress. We call these stress components the microstresses, and the planes on which they act the microplanes. In regard to the behavior on various microplanes and their interaction, we now introduce the following two basic hypotheses.

Hypothesis I.- The normal microstrain $e_{n}$, which governs the pro- 


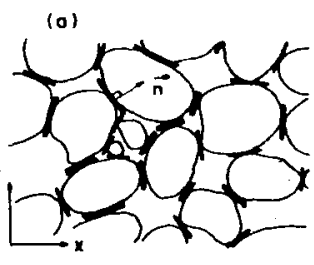

(b) $\sigma_{1}$

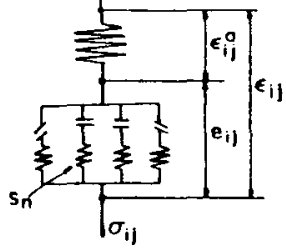

(e)
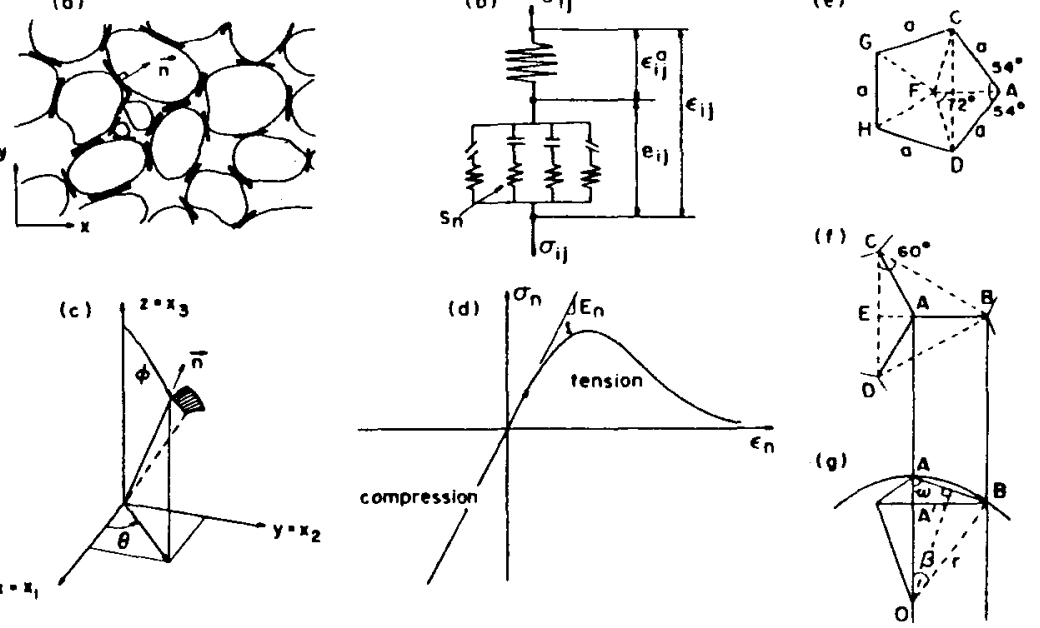

FIG. 1.-(a) Schematic Picture of Microstructure; (b) Present Rheological Model (c) Spherical Coordlnates; (d) Stress-Strain Relation for Microplane; $(e-g) \mathrm{Ge}$ ometry of Dodecahedron

gressive development of cracking on a microplane of any orientation, is equal to the resolved component of the macroscopic strain tensor $e_{i j}$ on the same plane, i.e.

$e_{n}=n_{i} n_{i} \mathcal{C}_{i}$

in which $n_{i}=$ direction cosines of the unit normal $i t$ of the microplane the latin subscripts refer to cartesian coordinates $x_{i}(i=1,2,3)$; and subscript repetition implies summation over 1,2 and 3 .

Hypothesis 11.- The stress relief due to all microcracks normal to $i$ is characterized by assuming that the microstress $s_{n}$ on a microplane of any orientation is a function of $e_{n}$ on the same plane [Fig. $\left.1(d)\right]$, i.e.

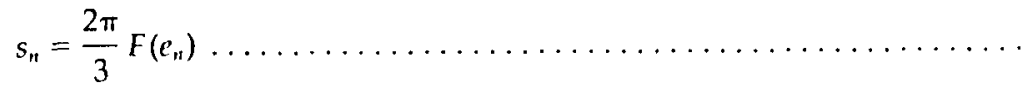

The factor $(2 \pi / 3)$ is introduced strictly for convenience, since later it will cancel out.

Hypothesis II is analogous to that made for shear microstresses and microstrains in the slip theory of plasticity, except that it deals with nor mal rather than shear components. Hypothesis I, however, is opposite In the previous theories, in which plasticity is described through the contributions from planes of various orientations in the material, it has been assumed that the microstructure is statically determinate rather than kinematically determinate (23), i.e., that the microstresses on all planes correspond to the same macroscopic stress.

There are two reasons for considering, in Hypothesis $\mathrm{I}$, that the microstructure is kinematically determinate. The first and more compelling reason is that the alternative, statically determinate models attempted at first always become unstable after the start of strain softening, as ı. spired from extensive computer simulations. Secondly, it seems that the hypothesis of kinematical determinacy reflects the microstructure of britthe aggregate materials better than the hypothesis of statical determinacy. Such materials consist of hard inclusions bound by a soft matrix. The stresses are obviously far from uniform, having sharp extremes in the thin contact layers of matrix between two aggregate pieces [Fig. 1(a)]. The microcracking in these thin contact layers, which may be imagined as the microplanes, makes the major contribution to inelastic strain. The deformations of these layers are determined chiefly by the relative displacements of the adjacent aggregate pieces, and since these relative displacements may be expected to approximately follow the smoothed macroscopic displacement field, the deformation of the thin contact layers between the aggregate pieces is approximately determined by the macroscopic strain, as assumed in Eq. 1.

In Hypothesis II, we neglect the shear stiffness on the microplanes, i.e., we assume the shear strains $e_{m}$ on the microplanes to take place freely and unrestricted. This feature simplifies mathematical modeling. It does not mean, however, that the material as a whole would have no shear stiffness, since shear deformations on any one plane are opposed by normal stiffness on planes inclined with respect to that plane.

In contrast to the well-known slip theory of plasticity, we include, in the stress-strain law for the microplanes (Eq. 2), not only the inelastic behavior but also the elastic behavior. This means that the curve $F\left(e_{n}\right)$ has a finite initial slope at $e_{n}=0$ [Fig. 1(d)]. Intuitively, the kinematic hypothesis $(\mathrm{Eq} .1)$ would probably be untrue if the elastic behavior were not included in Eq. 2. The alternative, where the microplanes exhibit no elastic behavior, i.e. the initial tangent of curve $F\left(e_{n}\right)$ is vertical, has also been carefully explored. The elastic response must then be represented as a strain added to strain $e_{i j}$ due to the microplane system, as in the slip theory of plasticity. This approach, however, appeared unworkable for the strain-softening range. A slowly declining strain-softening diagram could not be obtained, and stress dropped suddenly as soon as strain softening started. Moreover, computer simulations were very sensitive to the step size and appeared unstable, apparently because the strain in the microplanes could not be directly controlled in this alternative.

To sum up, it has been found in the course of this work that strain softening cannot be computationally simulated in a stable manner when: (1) The microplane system is constrained statically; or (2) the microplane system is constrained kinematically but is perfectly rigid initially. After eliminating these alternatives, we are thus left with Hypothesis I (Eq. 1), along with Eq. 2, which includes elastic stiffness, as the only simple and feasible hypothesis for the interaction within the microplane system

\section{incaemental Stiffness}

To determine the equilibrium conditions relating $\sigma_{i j}$ and $s_{n}$, we may consider the virtual work due to $\delta e_{i j}$. Thus 
$\delta W=\frac{4}{3} \pi \sigma_{i j} \delta e_{i j}=2 \int_{S} s_{n} \delta e_{n} f(\vec{n}) d S$

in which the factor $(4 \pi / 3)$ corresponds to integrating over a sphere of radius $1 ; S=$ surface of a unit hemisphere; and $d S=\sin \phi d \theta d \phi$ [Fig. $1(c)$ ]. Note that we do not need to integrate over the entire surface of the sphere, since the values of $\sigma_{n}$ and $e_{n}$ are equal at any two diametrically opposite points. Function $f(\vec{n})$ is the given normalized frequency distribution of microplanes of various orientations, characterizing the anisotropic properties of the material; $f(\vec{n}) \equiv 1$ represents isotropy, which we assume from now on for concrete. Substituting Eqs. 1 and 2 in Eq. 3 , we obtain

$\sigma_{i j} \delta e_{i j}=\int_{S} F\left(e_{n}\right) n_{i} n_{j} \delta e_{i j} d S$

Since this must hold for any $\delta e_{i j}$, we must have

$\sigma_{i j}=\int_{0}^{2 \pi} \int_{0}^{\pi / 2} F\left(e_{n}\right) n_{i} n_{j} \sin \phi d \phi d \theta$

According to Eq. 1

$d F\left(e_{n}\right)=F^{\prime}\left(e_{n}\right) d e_{n}=F^{\prime}\left(e_{n}\right) n_{k} n_{m} d e_{k m}$

and, thus, differentiation of Eq. 5 and substitution of Eq. 6 finally provide

$d \sigma_{i j}=D_{i j k m}^{c} d e_{k m}$

in which

$D_{i j k m}^{c}=\int_{0}^{2 \pi} \int_{0}^{\pi / 2} a_{i j k m} F^{\prime}\left(e_{n}\right) \sin \phi d \phi d \theta$, with $a_{i j k m}=n_{i} n_{j} n_{k} n_{m} \ldots \ldots \ldots$

$D_{i j k m}^{c}$ may be called the tangent stiffnesses of the microplane system. Note that the sequence of subscripts of $a_{i j k m}$ and of $D_{i j k m}^{c}$ is immaterial, and so the tensors $a_{i j k m}$ and $D_{i j k m}^{c}$ are fully symmetric and have only six independent values.

It is now important to realize that Hypothesis I implies a certain restriction on the elastic Poisson ratio $\nu^{\prime \prime}$ of the microplane system. To show it, assume that tensor $e_{i j}$ corresponds to uniaxial strain, i.e., $e_{z} \neq$ 0 , and $e_{x}=e_{y}=0\left(x, y\right.$ and $z$ correspond here to the axes $x_{1}, x_{2}$ and $\left.x_{3}\right)$ [Fig. $1(c)$ ], and calculate $\sigma_{z}, \sigma_{y}$. We note that $n_{z}=n_{3}=\cos \phi ; n_{y}=n_{2}$ $=\sin \phi \sin \theta ; n_{x}=n_{1}=\sin \phi \cos \theta ;$ and $\epsilon_{n}=\epsilon_{z} \cos ^{2} \phi ;$ and we consider that for small strains $F\left(\epsilon_{n}\right)=E_{n} \epsilon_{n}$, in which $E_{n}=F^{\prime}(0)<\infty$. Then, using Eq. 5, we obtain

$\sigma_{z}=\int_{0}^{2 \pi} \int_{0}^{\pi / 2} E_{n} \epsilon_{z} \cos ^{4} \phi \sin \phi d \phi d \theta=\frac{2 \pi}{5} E_{n} \epsilon_{z}$ $\sigma_{y}=\int_{0}^{2 \pi} \int_{0}^{\pi / 2} E_{n} \epsilon_{2}(\sin \phi \sin \theta)^{2} \cos ^{2} \phi \sin \phi d \phi d \theta=\frac{2 \pi}{15} E_{n} \epsilon_{z}$

Thus, $\sigma_{y} / \sigma_{z}=1 / 3$ and, because Hooke's law requires $\sigma_{y} / \sigma_{z}=\nu^{m} /(1-$ $\left.\nu^{m}\right)$, we see that Poisson's ratio $v^{m}$ is always $1 / 4$, as a consequence of Hypothesis I (Eq. 1). This is inapplicable to concrete, for which the Poisson ratio typically is about 0.18 .

The Poisson ratio could be adjusted to any value by including elastic shear stiffness for the microplanes, as has been done in a previous work (5). However, the formulation becomes considerably more complicated, especially due to the fact that there are infinitely many possible shear directions within each microplane. We may, however, adjust the value of $v^{m}$ by introducing additional elastic strain $\epsilon_{i j}^{a}$ so that the total strain $\epsilon_{i j}$ of the material is

$\epsilon_{i j}=e_{i j}+\epsilon_{i j}^{a}$

in which $e_{i j}=$ strain of the microplane system. This is visualized by the rheologic model in Fig. 1(b). The compliances corresponding to the additional elastic strain $\epsilon_{i j}^{a}$ may be considered in the well-known form:

$C_{i j k m}^{a}=\frac{1}{9 K^{a}} \delta_{i j} \delta_{k m}+\frac{1}{2 G^{a}}\left(\delta_{i k} \delta_{j m}-\frac{1}{3} \delta_{i j} \delta_{k m}\right)$

satisfying the condition of isotropy; $K^{a}$ and $G^{a}=$ additional bulk and shear moduli which cannot be less than the actual initial bulk and shear moduli, $K$ and $G$.

From the viewpoint of stability, we need $C_{i j k m}^{n}$ to be as stiff as possible, or else computer simulations of strain softening would not be stable, as explained earlier. Therefore, we choose either $1 / G^{a}=0$ or $1 / K^{a}=0$. Generally, if $1 / K^{a}>0$ and $1 / G^{a}=0$, the overall Poisson ratio $v$ is less than the Poisson ratio $v^{m}$ of the microplane system, determined above as 0.25 ; and if $1 / K^{a}=0$ and $1 / G^{a}>0, v$ is greater than $\nu^{m}$. For concrete, we need the former case, i.e., $1 / G^{a}=0$. Let us now determine the value of $K^{a}$ needed to achieve the desired Poisson ratio $\nu$. For this purpose we consider uniaxial stress $\sigma_{11}$, for which

$\epsilon_{11}=\frac{1}{E^{m}} \sigma_{11}+\frac{1}{3 K^{a}} \frac{\sigma_{11}}{3} ; \quad \sigma_{22}=\frac{-\nu^{m}}{E^{m}} \sigma_{11}+\frac{1}{3 K^{a}} \frac{\sigma_{11}}{3}$

in which $v^{m}=1 / 4$ and $E^{m}=2 \pi E_{n} / 5$. Since $\epsilon_{22}=-v \epsilon_{11}$, we obtain

$K^{a}=\frac{1+v}{9\left(\nu^{m}-v\right)} E^{m} \quad\left(\right.$ for $\left.\quad \nu \leq \nu^{m}, G^{a} \rightarrow \infty\right)$.

In view of Eq. 11 [and Fig. 1(b)], the incremental stress-strain relation may now be written as

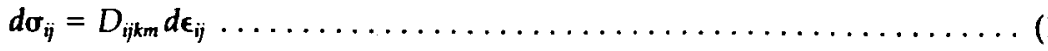

in which

$\left[D_{i, k m}\right]=\left[\left(D_{i, k m}^{X}\right)^{-1}+\left(C_{i, k m}^{a}\right)\right]^{-1}$ 
Here the brackets and parentheses refer to $6 \times 6$ square matrices formed from the tensor components.

Finally, we need to define the constitutive law for the microplanes, relating $\sigma_{n}$ to $\epsilon_{n}$. Our objective is to describe cracking all the way to complete fracture, at which $\sigma_{n}$ reduces to zero. Thus, $\sigma_{n}$ as a function of $\epsilon_{n}$ must first rise, then reach a maximum, and then gradually decline to zero. We choose the final zero value to be attained asymptotically, since we have no precise information on the final strain at which $\sigma_{n}=$ 0 , and since a smooth curve is convenient computationally. The following expressions are adopted [Fig. 1(d)\}:

$$
\begin{aligned}
& \text { for } \left.\quad \epsilon_{n}>0: \quad \sigma_{n}=E_{n} \epsilon_{n} \mathrm{e}^{-\left(k \epsilon_{n}^{p}\right)} \quad \text { (if } d \epsilon_{n} \geq 0\right) \ldots \ldots \ldots \ldots \ldots \ldots \ldots \ldots \ldots \ldots \ldots \ldots \ldots \ldots \ldots \ldots \ldots \ldots \ldots \ldots \ldots \ldots \ldots \ldots \ldots \ldots
\end{aligned}
$$

in which $E_{n}, k$ and $p=$ positive constants. Note that the slope and cur vature are continuous through the point $\epsilon_{n}=0$ if $p \geq 1$, and only the slope is continuous if $0 \leq p<1$.

\section{Numerical Integration on Surface of a Sphere}

Normally, the integral in Eq. 8 over the surface of a hemisphere has to be evaluated numerically. The numerical integration formula may be written in the form

$D_{i j k m}^{c} \simeq \sum_{n-1}^{N} w_{\alpha}\left[a_{i j k m} F^{\prime}\left(e_{n}\right)\right]_{0}$

in which $w^{\alpha}=$ weights (or coefficients); and $\alpha=1,2, \ldots, N=$ numbers of the numerical integration points on the hemisphere surface of radius 1 , defined by the unit vectors, $\vec{n}^{\alpha}$.

Since there are six independent incremental stiffnesses in Eq. 8, the numerical integration must be carried out six times for each point of the material where the stiffness is needed. In a finite element program, the numerical integration over a hemisphere must be repeated for all the finite elements and all the integration points within each element, and for all the loading steps. Obviously, it is important to use a very efficient numerical integration formula.

Carrying out the numerical integration by means of a rectangular mesh in the $(\theta \phi)$ plane is simple, but rather inefficient. One reason is that the integration points are wastefully crowded near the pole. More importantly, functions that are smooth on the spherical surface near the pole may be unsmooth in the $(\theta, \phi)$ plane. Therefore, optimal integration formulas should be constructed directly for the surface of the sphere. The greatest efficiency is achieved with a regular (uniform) distribution of the integration points over the spherical surface. Such a distribution is given either by the centroids of the faces of a regular polyhedron circumscribed to the sphere, or by the vertices of a dual regular polyhedron inscribed to the sphere. The regular polyhedron (Platonic solid) with the greatest possible number of faces is the icosahedron, having 20 faces (13). So, we cannot have, for a hemisphere, a numerical integration formula with more than $N=10$ regularly spaced points; see Fig. 2(a), in
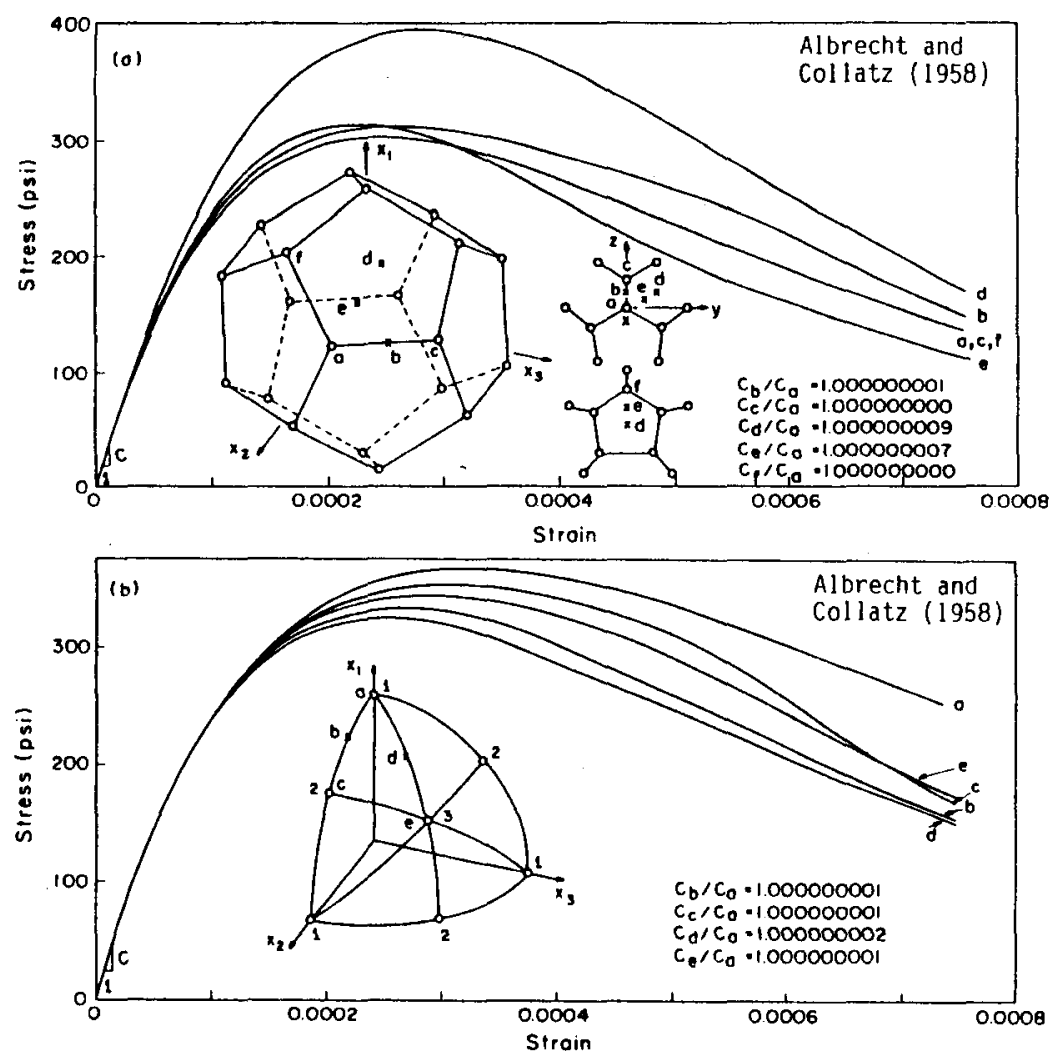

FIG. 2.-Response Curves for Unlaxial Stress of Various Orientations With Respect to Integration Points for Albrecht and Collatz's integration Formulas $(22,1,2)$ : (a) $2 \times 10$ Points; (b) $2 \times 13$ Points

which the integration points are pictured as the vertices of a dual dodecahedron. Such a numerical integration formula, which has equal weights, was given by Albrecht and Collatz (2). This formula is of the 5 th degree, which might seem to suffice for good accuracy, as it does for elastic behavior. Not for strain softening, however.

The accuracy of a numerical integration formula in mathematics is usually judged by the degree of the formula, representing the highest degree of the polynomial that is integrated by the formula exactly. But formulas of the same degree often show very different errors. To get a better idea of the accuracy of the integration formula, we propose the following test. The stress-strain curves calculated with the help of the numerical integration formula must remain nearly the same when the set of the integration points is arbitrarily rotated as a rigid body with respect to the material, while the applied stress or strain is not rotated. The maximum difference among the stress-strain curves for all such possible rotations is a measure of the error

Consider the calculation of the tensile stress-strain curve for uniaxial 

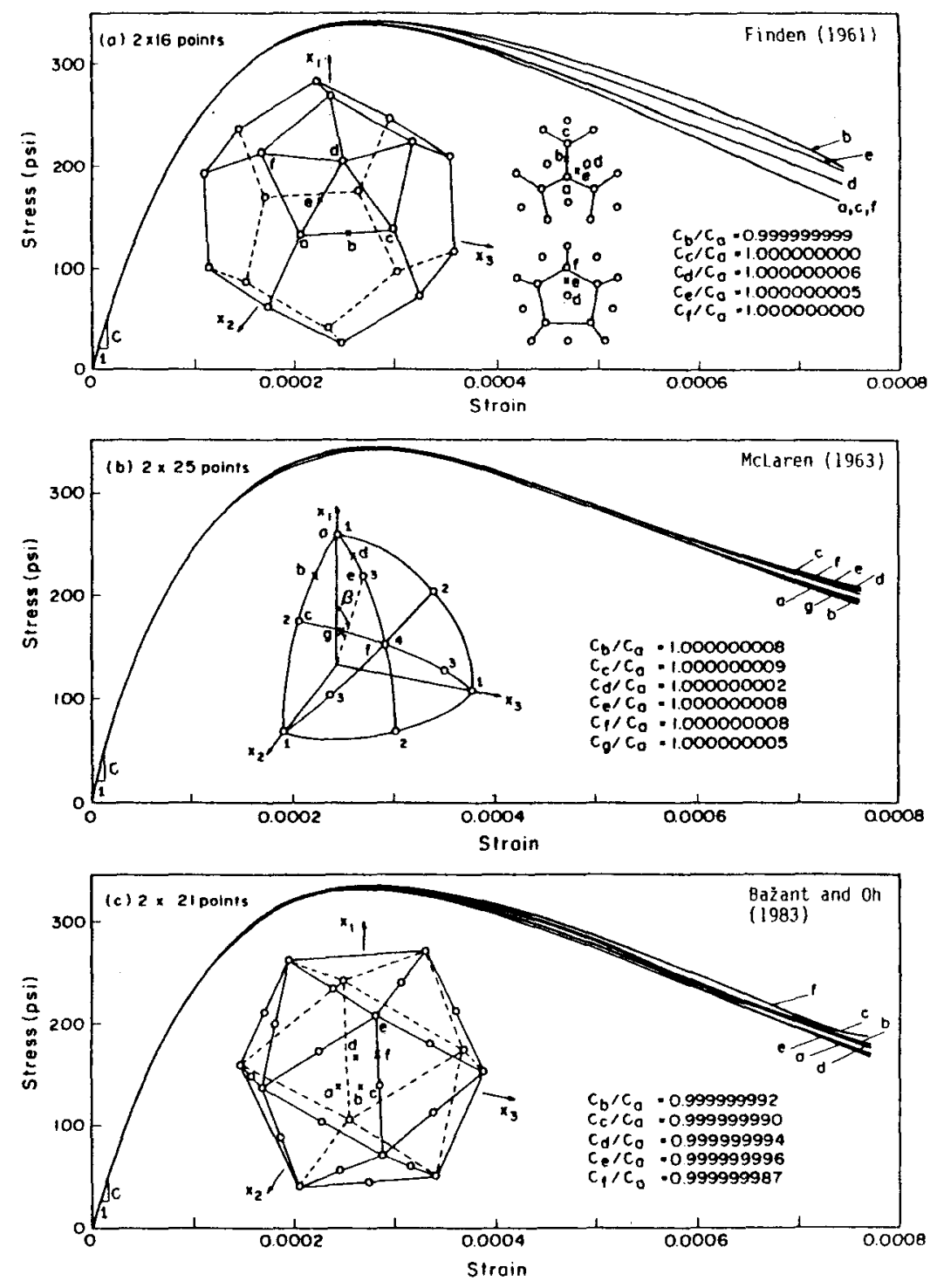

FIG. 3.-Response Curves for Unlaxial Stress of Various Orientations for Formulas of: (a) Finden (22); (b) McLaren (22); (c) Bažant-Oh (7)

stress. We specify the strain $\epsilon_{11}$ to increase in small steps $\Delta \epsilon_{11}$. For each such loading step, we evaluate the incremental stiffness matrix with the help of Eq. 19, and solve the increments $\Delta \sigma_{11}$ and all remaining $\Delta \epsilon_{i j}$ components from the conditions that all $\Delta \sigma_{i j}$ components except $\Delta \sigma_{11}$ must be zero. The calculations of the response curves for uniaxial tensile stress have been carried out using, in Eqs. $17-18, E_{11}=3,485,000$ psi; $k=6,280$; and $p=1$. The calculations are repeated for various orientations of axis $x_{1}$ of the uniaxial stress $\sigma_{11}$ relative to the set of integration points. In particular, the orientation coinciding with one integration point and other orientations between the integration points are used. Various $\sigma_{11}$ orientations considered are labeled in Figs. 2 and 3 as $a, b, c, \ldots$, and the corresponding response curves are labeled the same.

For Albrecht and Collatz's 10-point integration formula based on the faces of an icosahedron, the response curves for various $\sigma_{1 /}$ orientations are found to differ enormously from each other within the strain-softening range [Fig. 2(a)], even though their initial (elastic) slopes $C_{a}, C_{b}$, $C_{c}, \ldots$ are nearly the same for all orientations $a, b, c, \ldots$ las indicated in Fig. 2(a)]. Therefore, formulas of a higher degree, for which the spacing of integration points and the weights are nonuniform, must be used in the case of strain softening.

A number of numerical integration formulas for the surface of a sphere are listed in Stroud's book (22). Among these formulas, those which are not centrally symmetric (i.e., nonsymmetric with respect to the center of the sphere) have to be rejected when integration over only a hemisphere is of interest. The arrangements of the integration points for various applicable known formulas (22) are shown in Fig. 2 and Fig. 3(a-c), and their degrees are also indicated. Some of these formulas of higher
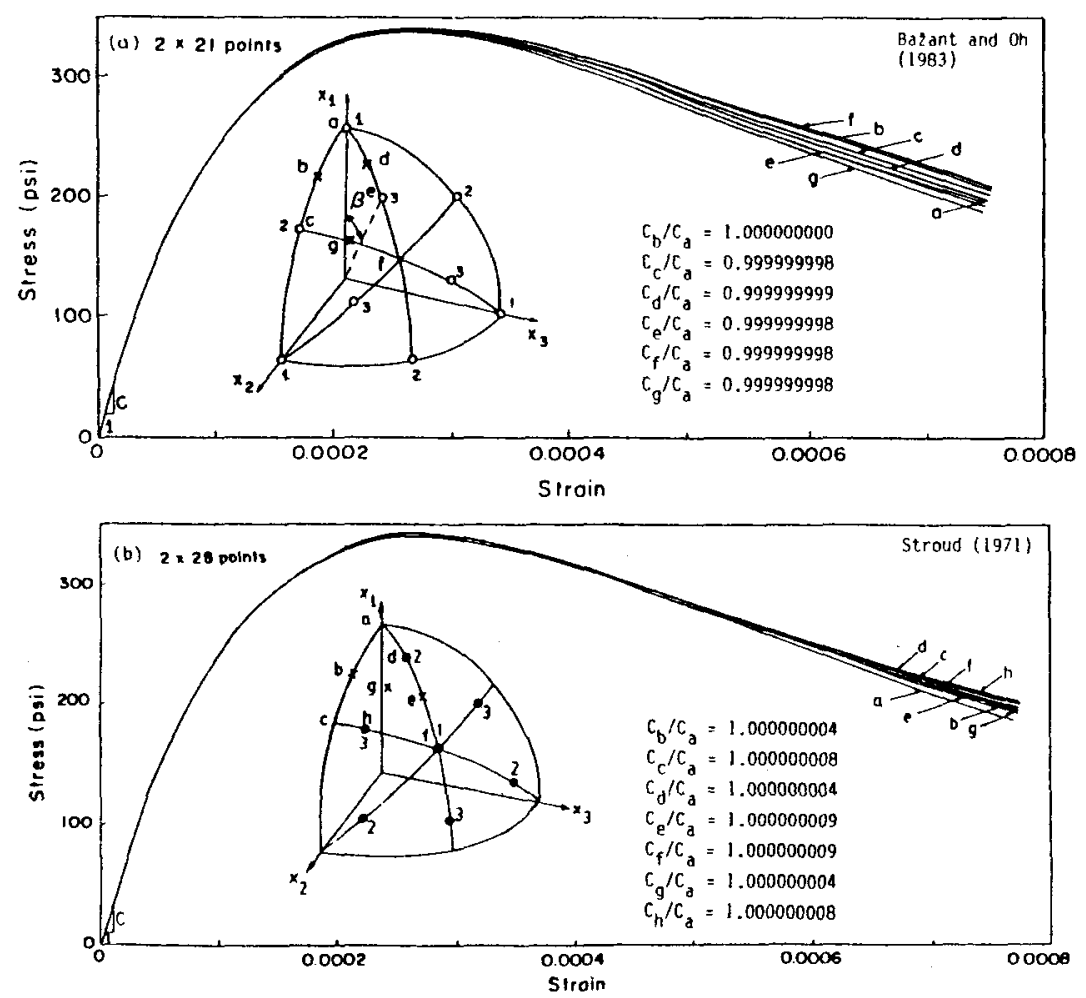

FIG. 4.-Response Curves for Unlaxial Stress of Varlous Orientations for Formulas of (a) Bażant-Oh (7); (b) Stroud (22) 
degree are based on subdivisions of a dodecahedron or a dual icosahedron [see, e.g., Finden's formula with $N=16$ in Fig. 3(a)], and some others are based on subdivisions of an octahedron; see, e.g., McLaren's formula with $N=25$ in Fig. $3(b)$ and Stroud's formula with $N=28$ in Fig. 4(b) for Albrecht and Collatz's formula (2), with $N=13$ in Fig. $2(b)$ which is, however, also too inaccurate for strain softening, as is clear from Fig. 2(b)].
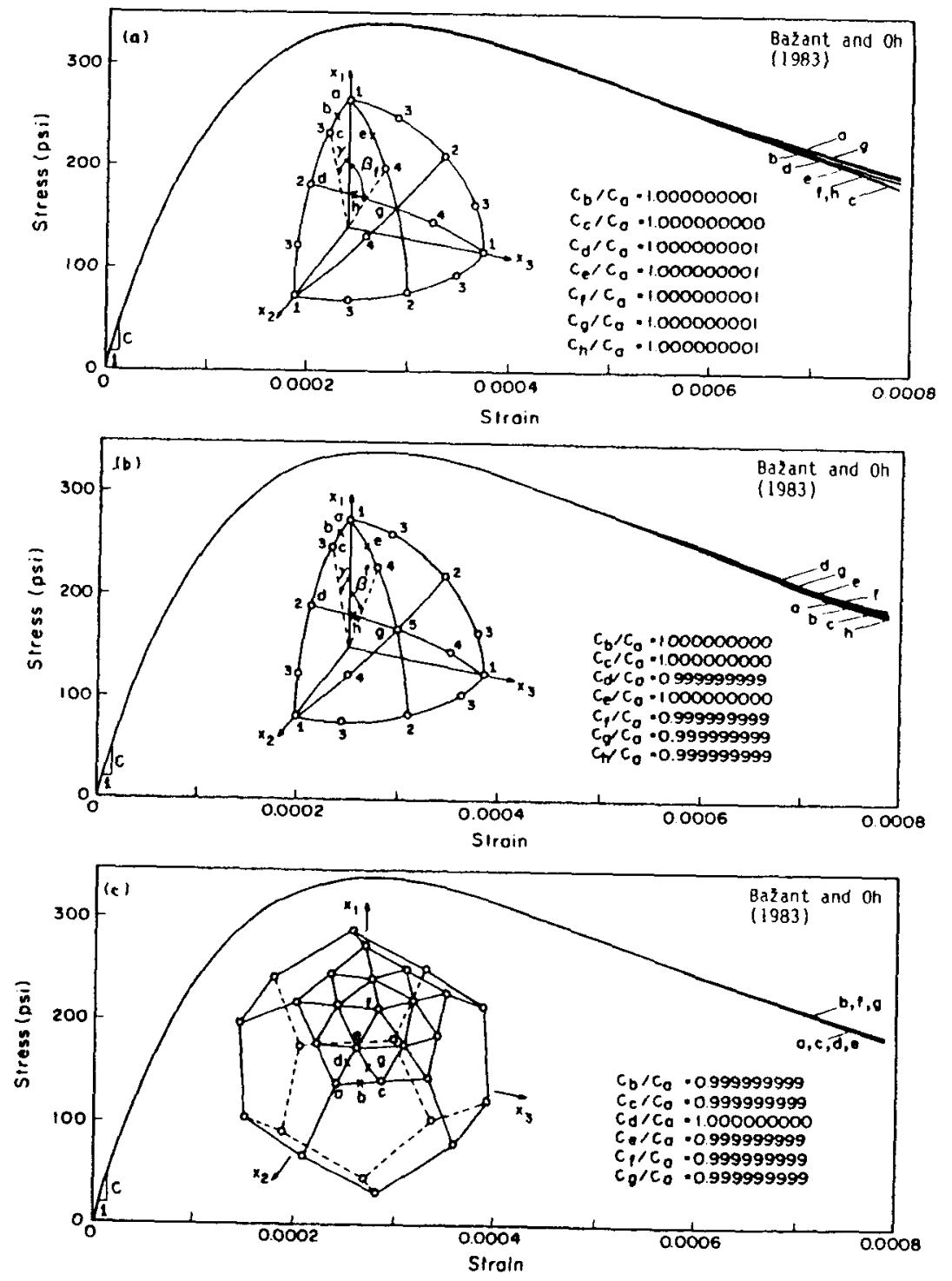

FIG. 5.-Response Curves for Uniaxial Stress of Various Orientations for New Bažant-Oh Formulas: (a) $2 \times 33$ Points; (b) $2 \times 37$ Points; (c) $2 \times 61$ Points
The aforementioned formulas with $N=25$ and $N=28$ are fully symmetric, i.e., symmetric with respect to all the three cartesian coordinate planes as well as all the six planes that contain one coordinate axis and form angles $45^{\circ}$ with the other two coordinate axes. This is advantageous when the strain and stress states exhibit orthogonal symmetries, as is the case for plane stress or plane strain. In such a case, a number of integration points may be omitted due to symmetry. For example, McLaren's formula with $N=25$ [Fig. 3(b)] requires function evaluation at only 16 points for the case of plane stress or plane strain, and Stroud's formula with $N=28$ [Fig. $4(b)$ ] requires it at only 14 points. The latter formula appears optimal for plane stress or plane strain (as well as axisymmetric states) if errors of about $\pm 3 \%$ are acceptable.

For McLaren's formula (Ref. 22, p. 301) with $N=25$, the points in the first octant [Fig. $3(b)]$ are $(1,0,0),(0,1,0)$ and $(0,0,1)$ with weights 9,216 / 725,$760 ;\left(c_{1}, c_{1}, 0\right),\left(c_{1}, 0, c_{1}\right)$ and $\left(0, c_{1}, c_{1}\right)$ with weights $16,384 / 725,760$; $\left(c_{2}, c_{2}, c_{2}\right)$ with weights 15,309/725,760; and $\left(c_{3}, c_{3}, c_{4}\right),\left(c_{3}, c_{4}, c_{3}\right)$ and $\left(c_{4}, c_{3}, c_{3}\right)$ with weights $14,641 / 725,760$, in which $c_{1}^{2}=1 / 2 ; c_{2}^{2}=1 / 3 ; c_{3}^{2}=1 / 11$ and $c_{4}^{2}=9 / 11$. For Stroud's formula (Ref. 22, p. 301) with $N=28$, the points in the first octant [Fig. $4(b)$ ] are $\left(c_{1}, c_{1}, c_{1}\right)$ with weights $9 / 560 ;\left(c_{2}, c_{3}, c_{3}\right)$, $\left(c_{3}, c_{2}, c_{3}\right)$ and $\left(c_{3}, c_{3}, c_{2}\right)$ with weights $(122+9 \sqrt{3}) / 6,720 ;\left(c_{4}, c_{5}, c_{5}\right),\left(c_{5}, c_{4}, c_{5}\right)$ and $\left(c_{5}, c_{5}, c_{4}\right)$ with weights $(122-9 \sqrt{3}) / 6,720$, in which $c_{1}^{2}=1 / 3 ; c_{2}^{2}=$ $(15+8 \sqrt{3}) / 33 ; c_{3}^{2}=(9-4 \sqrt{3}) / 33 ; c_{4}^{2}=(15-8 \sqrt{3}) / 33 ;$ and $c_{5}^{2}=(9+$ $4 \sqrt{3}) / 33$. The points and weights for the other octants are symmetric.

The response curves for various $\sigma_{11}$ orientations, as shown in Figs. $3(b)$

TABLE 1.-Direction Cosines and Weights for $2 \times 21$ Points [Degree 9, No Orthogonal Symmetrles, Fig. 3(c)]

\begin{tabular}{r|c|c|c|c}
\hline $\begin{array}{c}\alpha \\
(1)\end{array}$ & $\begin{array}{c}n_{i}^{\mathrm{o}} \\
(2)\end{array}$ & $\begin{array}{c}n_{i}^{\mathrm{o}} \\
(3)\end{array}$ & $\begin{array}{c}n_{3}^{\mathrm{a}} \\
(4)\end{array}$ & $\begin{array}{c}w_{r} \\
(5)\end{array}$ \\
\hline 1 & 0.187592474085 & 0 & 0.982246946377 & 0.0198412698413 \\
2 & 0.794654472292 & -0.525731112119 & 0.303530999103 & 0.0198412698413 \\
3 & 0.794654472292 & 0.525731112119 & 0.303530999103 & 0.0198412698413 \\
4 & 0.187592474085 & -0.850650808352 & -0.491123473188 & 0.0198412698413 \\
5 & 0.794654472292 & 0 & -0.607061998207 & 0.0198412698413 \\
6 & 0.187592474085 & 0.850650808352 & -0.491123473188 & 0.0198412698413 \\
7 & 0.577350269190 & -0.309016994375 & 0.755761314076 & 0.0253968253968 \\
8 & 0.577350269190 & 0.309016994375 & 0.755761314076 & 0.0253968253968 \\
9 & 0.934172358963 & 0 & 0.356822089773 & 0.0253968253968 \\
10 & 0.577350269190 & -0.809016994375 & -0.110264089708 & 0.0253968253968 \\
11 & 0.934172358963 & -0.309016994375 & -0.178411044887 & 0.0253968253968 \\
12 & 0.934172358963 & 0.309016994375 & -0.178411044887 & 0.0253968253968 \\
13 & 0.577350269190 & 0.809016994375 & -0.110264089708 & 0.0253968253968 \\
14 & 0.577350269190 & -0.5 & -0.645497224368 & 0.0253968253968 \\
15 & 0.577350269190 & 0.5 & -0.645497224368 & 0.0253968253968 \\
16 & 0.356822089773 & -0.809016994375 & 0.467086179481 & 0.0253968253968 \\
17 & 0.356822089773 & 0 & -0.934172358963 & 0.0253968253968 \\
18 & 0.356822089773 & 0.809016994375 & 0.467086179481 & 0.0253968253968 \\
19 & 0 & -0.5 & 0.866025403784 & 0.0253968253968 \\
20 & 0 & -0.5 & -0.866025403784 & 0.0253968253968 \\
21 & 0 & 1 & 0 & 0.0253968253968 \\
\hline \hline
\end{tabular}


and $4(b)$, indicate considerable improvement over those in Fig. 2(a). Based on the comparison of these curves, McLaren's formula with 25 points [Fig. 3(b)] appears to be sufficiently accurate for most practical purposes, and most efficient among the known formulas, although for plane stress or plane strain, Stroud's formula with $N=28$ appears even more efficient since it reduces to only 14 points.

The question of optimal numerical integration formulas for a hemisphere has been studied further (7), and some new formulas have been found. In the process, a new method of deriving the weights and optimal point locations has also been developed (7). This method is simpler than the usual procedure (22), in that it does not utilize the theory of orthogonal polynomials; instead, it uses the Taylor series expansions directly for the function values at the surface of the sphere, and relies on the power of the computer to find the weights and point locations for which the degree of the error is maximized and the coefficient of the truncation term is minimized. Using this computer approach, new formulas shown in Figs. 3(c), 4(a) and $5(a-c)$, with the weights and direction cosines of integration points listed in Tables $1-5$, have been developed (7).

Based on testing the responses to the uniaxial stress of various orientations, as indicated by the curves in Figs. 4(a) and 5, the new formulas with $N=33,37$ and 61 points appear to be superior to the existing centrally symmetric formulas listed by Stroud (22). The new 21-point

TABLE 2.-Direction Cosines and Weights for $2 \times 21$ Points [Degree 9, Orthogonal Symmetries, Fig. 4(a)]

\begin{tabular}{|c|c|c|c|c|}
\hline $\begin{array}{c}\alpha \\
(1)\end{array}$ & $\begin{array}{l}n_{1}^{n} \\
(2)\end{array}$ & $\begin{array}{l}n_{2}^{n} \\
(3) \\
\end{array}$ & $\begin{array}{l}n_{1}^{n} \\
\text { (4) }\end{array}$ & $\begin{array}{l}w \\
\text { (5) }\end{array}$ \\
\hline 1 & 1 & 0 & 0 & 0.0265214244093 \\
\hline 2 & 0 & 1 & 0 & 0.0265214244093 \\
\hline 3 & 0 & 0 & 1 & 0.0265214244093 \\
\hline 4 & 0.707106781187 & 0.707106781187 & 0 & 0.0199301476312 \\
\hline 5 & 0.707106781187 & -0.707106781187 & 0 & 0.0199301476312 \\
\hline 6 & 0.707106781187 & 0 & 0.707106781187 & 0.0199301476312 \\
\hline 7 & 0.707106781187 & 0 & -0.707106781187 & 0.0199301476312 \\
\hline 8 & 0 & 0.707106781187 & 0.707106781187 & 0.0199301476312 \\
\hline 9 & 0 & 0.707106781187 & -0.707106781187 & 0.0199301476312 \\
\hline 10 & 0.387907304067 & 0.387907304067 & 0.836095596749 & 0.0250712367487 \\
\hline 11 & 0.387907304067 & 0.387907304067 & -0.836095596749 & 0.0250712367487 \\
\hline 12 & 0.387907304067 & -0.387907304067 & 0.836095596749 & 0.0250712367487 \\
\hline 13 & 0.387907304067 & -0.387907304067 & -0.836095596749 & 0.0250712367487 \\
\hline 14 & 0.387907304067 & 0.836095596749 & 0.387907304067 & 0.0250712367487 \\
\hline 15 & 0.387907304067 & 0.836095596749 & -0.387907304067 & 0.0250712367487 \\
\hline 16 & 0.387907304067 & -0.836095596749 & 0.387907304067 & 0.0250712367487 \\
\hline 17 & 0.387907304067 & -0.836095596749 & -0.387907304067 & 0.0250712367487 \\
\hline 18 & 0.836095596749 & 0.387907304067 & 0.387907304067 & 0.0250712367487 \\
\hline 19 & 0.836095596749 & 0.387907304067 & -0.387907304067 & 0.0250712367487 \\
\hline 20 & 0.836095596749 & -0.387907304067 & 0.387907304067 & 0.0250712367487 \\
\hline 21 & 0.836095596749 & -0.387907304067 & -0.387907304067 & 0.0250712367487 \\
\hline
\end{tabular}

Note: $\beta=33.2699078510^{\circ}$ in Fig. $4(a)$
TABLE 3.-Direction Cosines and Weights for $2 \times 33$ Points [Degree 11, Orthogonal Symmetries, Flg. 5(a)]

\begin{tabular}{|c|c|c|c|c|}
\hline $\begin{array}{c}\alpha \\
(1) \\
\end{array}$ & $\begin{array}{l}n_{1}^{\mathrm{\alpha}} \\
\text { (2) }\end{array}$ & $\begin{array}{l}n_{2}^{\mathrm{n}} \\
\text { (3) }\end{array}$ & $\begin{array}{l}n_{3}^{\mathrm{a}} \\
(4)\end{array}$ & $\begin{array}{l}w_{\alpha} \\
\text { (5) }\end{array}$ \\
\hline 1 & 1 & 0 & 0 & 0.0098535399343 \\
\hline 2 & 0 & 1 & 0 & 0.0098535399343 \\
\hline 3 & 0 & 0 & 0 & 0.0098535399343 \\
\hline 4 & 0.707106781187 & 0.707106781187 & 0 & 0.0162969685886 \\
\hline 5 & 0.707106781187 & -0.707106781187 & 0 & 0.0162969685886 \\
\hline 6 & 0.707106781187 & 0 & 0.707106781187 & 0.0162969685886 \\
\hline 7 & 0.707106781187 & 0 & -0.707106781187 & 0.0162969685886 \\
\hline 8 & 0 & 0.707106781187 & 0.707106781187 & 0.0162969685886 \\
\hline 9 & & 0.707106781187 & -0.707106781187 & 0.0162969685886 \\
\hline 10 & 0.933898956394 & 0.357537045978 & 0 & 0.0134788844008 \\
\hline 11 & 0.933898956394 & -0.357537045978 & 0 & 0.0134788844008 \\
\hline 12 & 0.357537045978 & 0.933898956394 & 0 & 0.0134788844008 \\
\hline 13 & 0.357537045978 & -0.933898956394 & 0 & 0.0134788844008 \\
\hline 14 & 0.933898956394 & 0 & 0.357537045978 & 0.0134788844008 \\
\hline 15 & 0.933898956394 & 0 & -0.357537045978 & 0.0134788844008 \\
\hline 16 & 0.357537045978 & 0 & 0.933898956394 & 0.0134788844008 \\
\hline 17 & 0.357537045978 & 0 & -0.933898956394 & 0.0134788844008 \\
\hline 18 & 0 & 0.933898956394 & 0.357537045978 & 0.0134788844008 \\
\hline 19 & 0 & 0.933898956394 & -0.357537045978 & 0.0134788844008 \\
\hline 20 & 0 & 0.357537045978 & 0.933898956394 & 0.0134788844008 \\
\hline 21 & 0 & 0.357537045978 & -0.933898956394 & 0.0134788844008 \\
\hline 22 & 0.437263676092 & 0.437263676092 & 0.785875915868 & 0.0175759129880 \\
\hline 23 & 0.437263676092 & 0.437263676092 & -0.785875915868 & 0.0175759129880 \\
\hline 24 & 0.437263676092 & -0.437263676092 & 0.785875915868 & 0.0175759129880 \\
\hline 25 & 0.437263676092 & -0.437263676092 & -0.785875915868 & 0.0175759129880 \\
\hline 26 & 0.437273676092 & 0.785875915868 & 0.437263676092 & 0.0175759129880 \\
\hline 27 & 0.437263676092 & 0.785875915868 & -0.437263676092 & 0.0175759129880 \\
\hline 28 & 0.437263676092 & -0.785875915868 & 0.437263676092 & 0.0175759129880 \\
\hline 29 & 0.437263676092 & -0.785875915868 & -0.437263676092 & 0.0175759129880 \\
\hline 30 & 0.785875915868 & 0.437263676092 & 0.437263676092 & 0.0175759129880 \\
\hline 31 & 0.785875915868 & 0.437263676092 & -0.437263676092 & 0.0175759129880 \\
\hline 32 & 0.785875915868 & -0.437263676092 & 0.437263676092 & 0.0175759129880 \\
\hline 33 & 0.785875915868 & -0.437263676092 & -0.437263676092 & 0.0175759129880 \\
\hline
\end{tabular}

formula in Fig. 4(a) shows about the same magnitude of error at the end of the curve as the 25-point McLaren's formula, and (in absence of planestress symmetry) it seems to represent the most efficient formula for this degree of accuracy ( $\pm 3 \%$ of maximum stress), which should suffice for many practical applications.

Note that the degree of the formula is not very indicative of the accuracy based on the test with various $\sigma_{11}$-directions. The formulas with $N=33$ and 37 appear to be approximately equally accurate, even though their degrees are 11 and 13, respectively. The new formulas with $N=$ 21 [Figs. 3(c), 4(a)] perform much better than Finden's formula with $N$ $=16$ [Fig. 3(a)], even though they are all of the 9th degree.

The foregoing calculations of the response curves using numerical in- 
TABLE 4.-Direction Cosines and Weights for $2 \times 37$ Points [Degree 13, Orthogonal Symmetries, Fig. $5(b)$ ]

\begin{tabular}{|c|c|c|c|c|}
\hline $\begin{array}{c}\alpha \\
\text { (1) }\end{array}$ & $\begin{array}{l}n_{1}^{\alpha} \\
(2) \\
\end{array}$ & $\begin{array}{l}n_{2}^{\sigma} \\
\text { (3) }\end{array}$ & $\begin{array}{r}n_{3}^{\alpha} \\
(4) \\
\end{array}$ & $\begin{array}{l}w_{a} \\
(5)\end{array}$ \\
\hline 1 & 1 & 0 & 0 & 0.0107238857303 \\
\hline 2 & 0 & 1 & 0 & 0.0107238857303 \\
\hline 3 & 0 & 0 & 0 & 0.0107238857303 \\
\hline 4 & 0.707106781187 & 0.707106781187 & 0 & 0.0211416095198 \\
\hline 5 & 0.707106781187 & -0.707106781187 & 0 & 0.0211416095198 \\
\hline 6 & 0.707106781187 & 0 & 0.707106781187 & 0.0211416095198 \\
\hline 7 & 0.707106781187 & 0 & -0.707106781187 & 0.0211416095198 \\
\hline 8 & 0 & 0.707106781187 & 0.707106781187 & 0.0211316095198 \\
\hline 9 & 0 & 0.707106781187 & -0.707106781187 & 0.0211416095198 \\
\hline 10 & 0.951077869651 & 0.308951267775 & 0 & 0.0053550559084 \\
\hline 11 & 0.951077869651 & -0.308951267775 & 0 & 0.0053550559084 \\
\hline 12 & 0.308951267775 & 0.951077869651 & 0 & 0.0053550559084 \\
\hline 13 & 0.308951267775 & -0.951077869651 & 0 & 0.0053550559084 \\
\hline 14 & 0.951077869651 & 0 & 0.308951267775 & 0.0053550559084 \\
\hline 15 & 0.951077869651 & 0 & -0.308951267775 & 0.0053550559084 \\
\hline 16 & 0.308951267775 & 0 & 0.951077869651 & 0.0053550559084 \\
\hline 17 & 0.308951267775 & 0 & -0.951077869651 & 0.0053550559084 \\
\hline 18 & 0 & 0.951077869651 & 0.308951267775 & 0.0053550559084 \\
\hline 19 & 0 & 0.951077869651 & -0.308951267775 & 0.0053550559084 \\
\hline 20 & 0 & 0.308951267775 & 0.951077869651 & 0.0053550559084 \\
\hline 21 & 0 & 0.308951267775 & -0.951077869651 & 0.0053550559084 \\
\hline 22 & 0.335154591939 & 0.335154591939 & 0.880535518310 & 0.0167770909156 \\
\hline 23 & 0.335154591939 & 0.335154591939 & -0.880535518310 & 0.0167770909156 \\
\hline 24 & 0.355154591939 & -0.335154591939 & 0.880535518310 & 0.0167770909156 \\
\hline 25 & 0.335154591939 & -0.335154591939 & -0.880535518310 & 0.0167770909156 \\
\hline 26 & 0.335154591939 & 0.880535518310 & 0.335154591939 & 0.0167770909156 \\
\hline 27 & 0.335154591939 & 0.880535518310 & -0.335154591939 & 0.0167770909156 \\
\hline 28 & 0.335154591939 & -0.880535518310 & 0.335154591939 & 0.0167770909156 \\
\hline 29 & 0.335154591939 & -0.880535518310 & -0.335154591939 & 0.0167770909156 \\
\hline 30 & 0.880535518310 & 0.335154591939 & 0.335154591939 & 0.0167770909156 \\
\hline 31 & 0.880535518310 & 0.335154591939 & -0.335154591939 & 0.0167770909156 \\
\hline 32 & 0.880535518310 & -0.335154591939 & 0.335154591939 & 0.0167770909156 \\
\hline 33 & 0.880535518310 & -0.335154591939 & -0.335154591939 & 0.0167770909156 \\
\hline 34 & 0.577350269190 & 0.577350269190 & 0.577350269190 & 0.0188482309508 \\
\hline 35 & 0.577350269190 & 0.577350269190 & -0.577350269190 & 0.0188482309508 \\
\hline 36 & 0.577350269190 & -0.577350269190 & 0.577350269190 & 0.0188482309508 \\
\hline 37 & 0.577350269190 & -0.577350269190 & -0.577350279190 & 0.0188482309508 \\
\hline
\end{tabular}

tegration formulas of increasing numbers of points confirm that convergence does indeed occur even in the strain-softening region. This would not be clear without this demonstration, and, in fact, for other models, e.g., the statically constrained microplane system, convergence does not take place.

\section{Procedure of Analysis and Comparison with Test Data}

The numerical algorithm used in the aforementioned calculations of
TABLE 5.-Direction Cosines and Welghts for $2 \times 61$ Polnts [Degree 13, No Orthogonal Symmetrles, Fig. 5(c)]

\begin{tabular}{|c|c|c|c|c|}
\hline $\begin{array}{c}\alpha \\
(1) \\
\end{array}$ & $\begin{array}{l}n_{1}^{\alpha} \\
(2)\end{array}$ & $\begin{array}{r}n_{2}^{\alpha} \\
(3) \\
\end{array}$ & $\begin{array}{l}n_{3}^{\alpha} \\
(4)\end{array}$ & $\begin{array}{l}w_{c} \\
(5)\end{array}$ \\
\hline 1 & 1 & 0 & 0 & 0.0079584420468 \\
\hline 2 & 0.745355992500 & 0 & 0.6666666666667 & 0.0079584420468 \\
\hline 3 & 0.745355992500 & -0.577350279190 & -0.333333333333 & 0.0079584420468 \\
\hline 4 & 0.745355992500 & 0.577350269190 & -0.33333333333333 & 0.0079584420468 \\
\hline 5 & 0.333333333333 & 0.577350279190 & 0.745355992500 & 0.0079584420468 \\
\hline 6 & 0.333333333333 & -0.577350269190 & 0.745355992500 & 0.0079584420468 \\
\hline 7 & 0.333333333333 & -0.934172358963 & 0.127322003750 & 0.0079584420468 \\
\hline 8 & 0.333333333333 & -0.356822089773 & -0.872677996250 & 0.0079584420468 \\
\hline 9 & 0.333333333333 & 0.356822089773 & -0.872677996250 & 0.0079584420468 \\
\hline 10 & 0.333333333333 & 0.934172358963 & 0.127322003750 & 0.0079584420468 \\
\hline 11 & 0.794654472292 & -0.525731112119 & 0.303530999103 & 0.0105155242892 \\
\hline 12 & 0.794654472292 & 0 & -0.607061998207 & 0.0105155242892 \\
\hline 13 & 0.794654472292 & 0.525731112119 & 0.303530999103 & 0.0105155242892 \\
\hline 14 & 0.187592474085 & 0 & 0.982246946377 & 0.0105155242892 \\
\hline 15 & 0.187592474085 & -0.850650808352 & -0.491123473188 & 0.0105155242892 \\
\hline 16 & 0.187592474085 & 0.850650808352 & -0.491123473188 & 0.0105155243892 \\
\hline 17 & 0.934172358963 & 0 & 0.356822089773 & 0.0100119364272 \\
\hline 18 & 0.934172358963 & -0.309016994375 & -0.178411044887 & 0.0100119364272 \\
\hline 19 & 0.934172358963 & 0.309016994375 & -0.178411044887 & 0.0100119364272 \\
\hline 20 & 0.577350269190 & 0.309016994375 & 0.755761314076 & 0.0100119364272 \\
\hline 21 & 0.577350269190 & -0.309016994375 & 0.755761314076 & 0.0100119364272 \\
\hline 22 & 0.577350269190 & -0.809016994375 & -0.110264089708 & 0.0100119364272 \\
\hline 23 & 0.577350269190 & -0.5 & -0.645497224368 & 0.0100119364272 \\
\hline 24 & 0.577350269190 & 0.5 & -0.645497224368 & 0.0100119364262 \\
\hline 25 & 0.577350269190 & 0.809016994375 & -0.110264089708 & 0.0100119364272 \\
\hline 26 & 0.356822089773 & -0.809016994375 & 0.467086179481 & 0.0100119364272 \\
\hline 27 & 0.356822089773 & 0 & -0.934172358963 & 0.0100119364272 \\
\hline 28 & 0.356822089773 & 0.809016994375 & 0.467086179481 & 0.0100119364272 \\
\hline 29 & 0 & 0.5 & 0.866025403784 & 0.0100119364272 \\
\hline 30 . & 0 & -1 & 0 & 0.0100119364272 \\
\hline 31 & 0 & 0.5 & -0.866025403784 & 0.0100119364272 \\
\hline 32 & 0.947273580412 & -0.277496978165 & 0.160212955043 & 0.0069047795797 \\
\hline 33 & 0.812864676392 & -0.277496978165 & 0.512100034157 & 0.0069047795797 \\
\hline 34 & 0.595386501297 & -0.582240127941 & 0.553634669695 & 0.0069047795797 \\
\hline 35 & 0.595386501297 & -0.770581752342 & 0.227417407053 & 0.0069047795797 \\
\hline 36 & 0.812864676392 & -0.582240127941 & -0.015730584514 & 0.0069047795797 \\
\hline 37 & 0.492438766306 & -0.753742692223 & -0.435173546254 & 0.0069047795797 \\
\hline 38 & 0.274960591212 & -0.942084316623 & -0.192025554687 & 0.0069047795797 \\
\hline 39 & -0.076926487903 & -0.942084316623 & -0.326434458707 & 0.0069047795797 \\
\hline 40 & -0.076926487903 & -0.753742692223 & -0.652651721349 & 0.0069047795797 \\
\hline 41 & 0.274960591212 & -0.637341166847 & -0.719856173359 & 0.0069047795797 \\
\hline 42 & 0.947273580412 & 0 & -0.320425910085 & 0.0069047795797 \\
\hline 43 & 0.812864676392 & -0.304743149777 & $-0.49636944^{\circ} 643$ & 0.0069047795797 \\
\hline 44 & 0.595386501297 & -0.188341624401 & -0.781052076747 & 0.0069047795797 \\
\hline 45 & 0.595386501297 & 0.188341624401 & -0.781052076747 & 0.0069047794797 \\
\hline 46 & 0.812864676392 & 0.304743149777 & -0.496369449643 & 0.0069047795797 \\
\hline 47 & 0.492438766306 & 0.753742692223 & -0.435173546254 & 0.0069047795797 \\
\hline 48 & 0.274960591212 & 0.637341166847 & -0.719856173359 & 0.0069047795797 \\
\hline
\end{tabular}




\begin{tabular}{c|r|r|r|c}
\hline$(1)$ & $(2)$ & \multicolumn{1}{c|}{$(3)$} & \multicolumn{1}{c}{$(4)$} & \multicolumn{1}{c}{$(5)$} \\
\hline 49 & -0.076926487903 & 0.753742692223 & -0.652651721349 & 0.0069047795797 \\
50 & -0.076926487903 & 0.942084316623 & -0.326434458707 & 0.0069047795797 \\
51 & 0.274960591212 & 0.942084316623 & -0.192025554687 & 0.0069047795797 \\
52 & 0.947273580412 & 0.277496978165 & 0.160212955043 & 0.0069047795797 \\
53 & 0.812864676392 & 0.582240127941 & -0.015730584514 & 0.0069047795797 \\
54 & 0.595386501297 & 0.770581752342 & 0.227417407053 & 0.0069047795797 \\
55 & 0.595386501297 & 0.582240127941 & 0.553634669695 & 0.0069047795797 \\
56 & 0.812864676392 & 0.277496978165 & 0.512100034157 & 0.0069047795797 \\
57 & 0.492438766306 & 0 & 0.870347092509 & 0.0069047795797 \\
58 & 0.274960591212 & 0.304743149777 & 0.911881728046 & 0.0069047795797 \\
59 & -0.076926487903 & 0.188341624401 & 0.979086180056 & 0.0069047795797 \\
60 & -0.076926487903 & -0.188341624401 & 0.979086180056 & 0.0069047795797 \\
61 & 0.274960591212 & -0.304743149777 & 0.911881728046 & 0.0069047795797 \\
\hline \hline
\end{tabular}

tensile response curves and also in the fitting of test data is as follows:

1. Determine $e_{n}^{(a)}$ from Eqs. 1 and 2 for the directions of all integration points $\alpha=1, \ldots, N$. In the first iteration of the loading step, use $\epsilon_{i j}$ for the end of the previous step, and in subsequent iterations use the value of $\epsilon_{i j}$ determined for the mid-step in the previous iteration. In structural analysis, repeat this for all finite elements and for all integration points within each finite element.

2. For all directions $n^{(\kappa)}$, evaluate $F^{\prime}\left(e_{n}\right)$ for use in Eq. 8. Also check whether unloading occurs in this direction, as indicated by violation of the condition $s_{n} \Delta e_{n} \geq 0$. If violated, replace $F^{\prime}\left(e_{n}\right)$ with the unloading stiffness, which may be approximately taken either as $E_{n}$ or according to the curve in Fig. 5 (for better expressions for unloading after strain softening, see Refs. 6 and 19).

3. Evaluate $D_{i, k m}^{r}$ from Eq. 19 and $D_{1, k m}$ from Eq. 16. In structural analysis, repeat this for all elements and all integration points in each element.

4. When solving stress-strain curves, calculate the increments of unknown stresses and unknown strains from Eq. 15. In structural analysis, solve (by the finite element method) the increments of nodal displacements from the given load increments, and subsequently calculate the increments of $\epsilon_{i j}$ and $\sigma_{i j}$ for all elements and all integration points in each element.

5. Advance to the next iteration of the same loading step, or advance to the next loading step.

In simulating uniaxial tensile loading of concrete, the unloading stressstrain curve for the microplane is not important since the only unloading occurs at moderate compressive stresses, for which a perfectly elastic (straight) unloading may be assumed.

The present model can be calibrated by comparison with direct tensile tests which cover the strain-softening response. Such tests, which can be carried out in a very stiff testing machine and on sufficiently small test specimens, have been performed by Evans and Marathe (14) as well
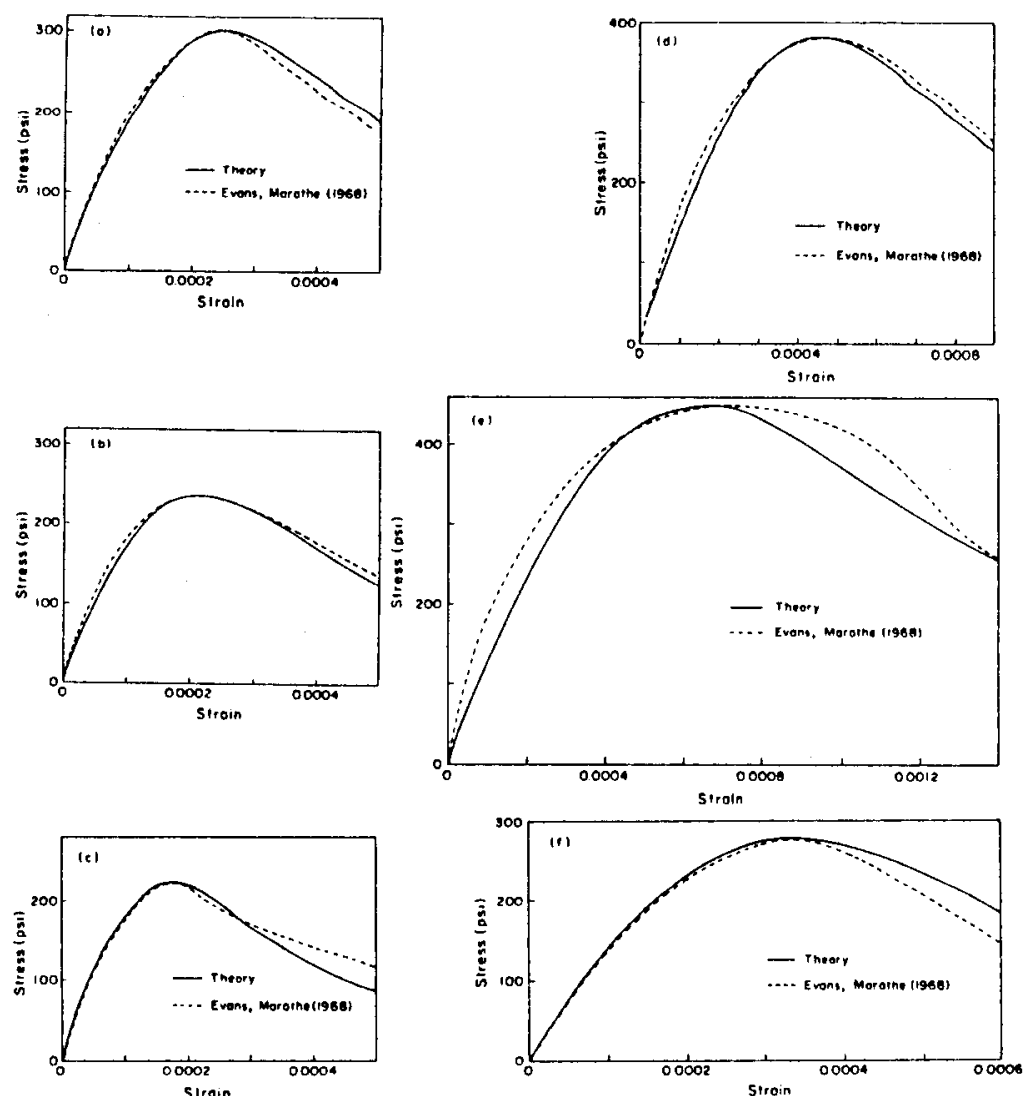

FIG. 6.-Best Fits of Present Theory to Test Data of Evans and Marathe (1968)

as others $(15,16,19,20)$. Optimal values of the three parameters of the model, $E_{n}, k$ and $p$, have been found in order to achieve the best fits of the data of Evans and Marathe. The fits are shown as the solid lines in Figs. $6(a-f)$, and the data are shown as the dashed lines. For all cases, $p=2$ was fixed, being nearly optimum. The values of $E_{n}$ for concretes $1,2, \ldots, 6$ tested were $2,100,1,876,2,149,1,433,1,194$ and $1,433 \mathrm{ksi}(1$ $\mathrm{ksi}=6,895 \mathrm{kPa}$ ), and those of $10^{-6} \mathrm{k}$ were $19.9,26.6,40.2,5.98,2.98$ and 11.1 , respectively. As is seen, satisfactory agreement with the test data can be easily achieved. A real test for the theory would, of course come only when tensile tests under rotating principal stress directions can be carried out.

Note that in our theory we have only two parameters, $E_{n}$ and $k$, to determine by fitting test data. A trial-and-error approach is sufficient for that.

\section{Application to General Loading Histories}

The preceding representation of tests can, of course, be achieved with 

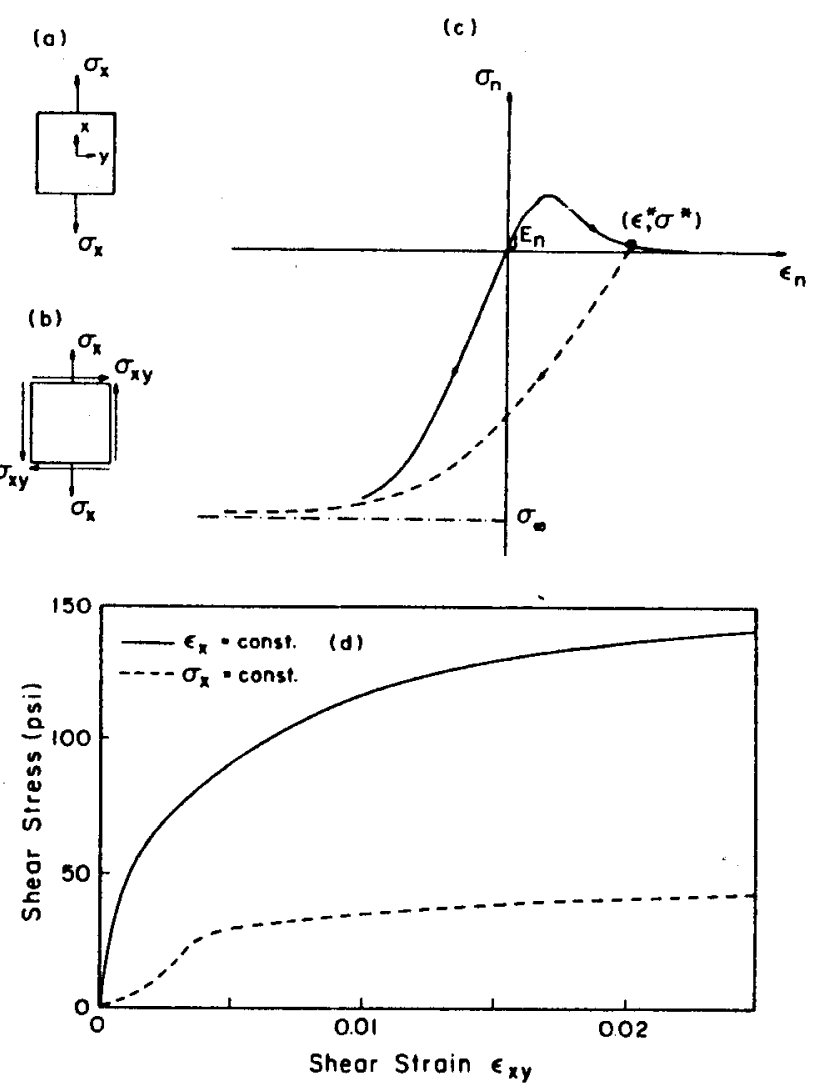

FIG. 7.- ( $a$ and $b)$ Unlaxlal Stress Followed by Shear Stress; $(c)$ Assumed Unloading Behavior for Microplane; (d) Calculated Responses for Constant $\epsilon_{x}$ or Constant $\sigma_{x}$

simple formulas, but this is not where the value of the theory lies. Rather, it is the fact that our model is incremental, path-dependent and tensorially invariant. Therefore, no fundamental principles of continuum me. chanics are violated when the present theory is applied to loading histories that are nonproportional, or during which the principal stress directions rotate. This is not the case for the existing simple descriptions of strain softening in tension.

Since no test data for tensile nonproportional loadings exist, we will at least show some predictions to demonstrate a typical capability of the theory. At first, we consider that uniaxial tension $\sigma_{x}$ is applied, increas ing the axial strain into the strain-softening range up to the point where the axial stress is reduced to one-half the previous peak. Subsequently, we keep either stress $\sigma_{x}$ or strain $\epsilon_{x}$ constant, and apply shear loading $\epsilon_{x y}$ [Fig. $7(a-b)$ ]. This type of loading is of interest, e.g., for seismic analysis of reinforced concrete walls of nuclear containments, which may be partially cracked in the horizontal direction before earthquake causes horizontal shear. Other typical examples of such loading histories may be found in blast loadings. These situations have thus far been analyzed under the assumption that continuous horizontal cracks exist, but in practice the cracking would usually be only partial before the shear is applied.

Carrying out the calculations, one finds that some of the microplanes are subjected for these histories to unloading from a tensile stress state that is possibly located well beyond the peak stress point. For such unloading, the assumption of elastic unloading (slope $E_{n}$ ) is probably too simplistic. Based on a separate study (6), in which the present model was used to fit the test data from the literature on shear loading of continuous cracks in concrete, it appears that the unloading behavior on the microplanes can be approximately described by the curve

$\sigma_{n}=-c_{1}+c_{2} \tan ^{-1}\left[c_{3}\left(\epsilon_{n}-c_{4}\right)\right]$

in which

$c_{2}=0.334 \sigma_{\infty}\left(1+1.38 e^{-400 \epsilon^{*}}\right) ; \quad c_{3}=\frac{0.07}{c_{2}}\left(1+13.29 e^{-14 \epsilon^{\cdot 025}}\right) E_{n} ；$

$c_{1}=\sigma_{\infty}-c_{2} \frac{\pi}{2} ; \quad c_{4}=\epsilon^{*}-\frac{1}{c_{3}} \tan \frac{\sigma^{*}+c_{1}}{c_{2}} ;$

$\sigma_{\infty}=\frac{1.86+15 \times 10^{5} \epsilon^{*^{3}}}{1+15 \times 10^{5} \delta^{*^{3}}} 0.6 f_{c}^{\prime}$

and $E_{n}=2,100,000 \mathrm{psi} ; k=0.199 \times 10^{8} ; p=2 ; f_{c}^{\prime}=2,533 \mathrm{psi} ; \sigma^{*}$ and $\epsilon^{*}$
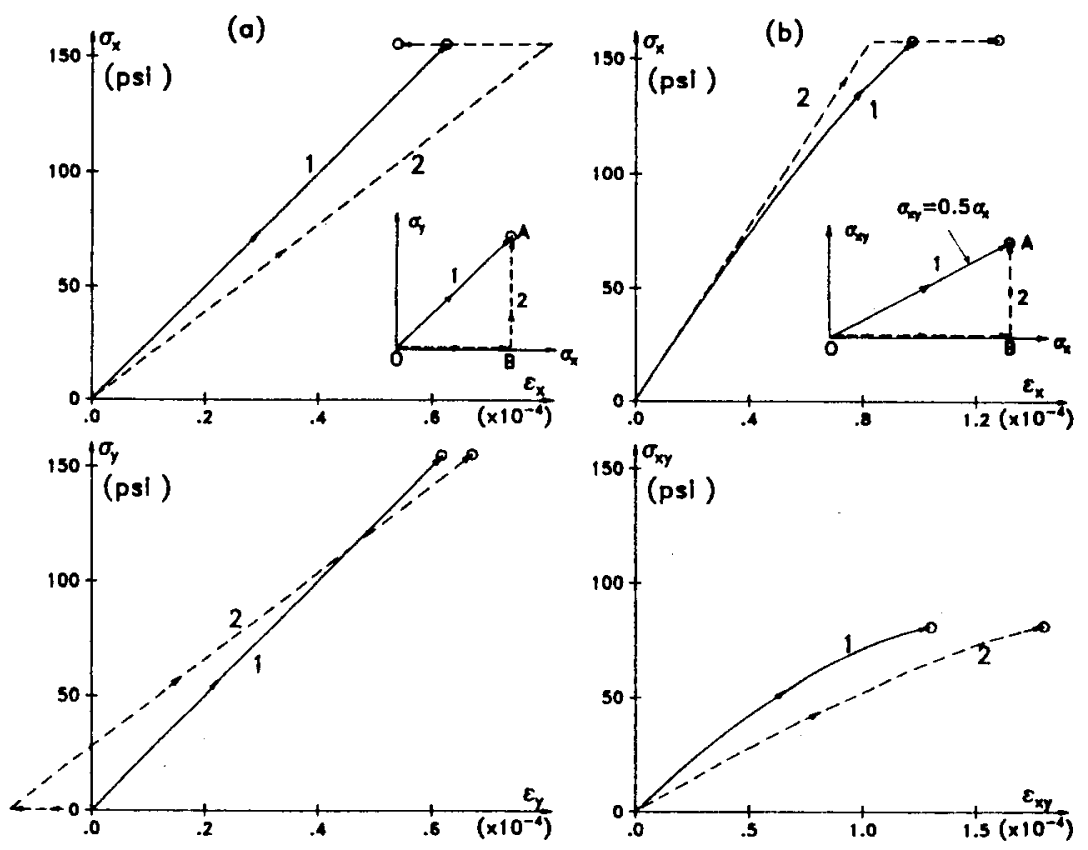

FIG. 8.-Path Dependence for Loading Paths Leading to Same FInal Stress State 
denote the stress and strain at the point where loading reverses to unloading; and $\sigma_{\infty}=$ parameter having the meaning of an asymptotic value in compression; see Fig. 7(c).

The calculated response curves for the two aforementioned loading histories are shown in Fig. $7(d)$. Note the tremendous difference be tween the curves for constant normal stress and constant normal strain

If there is no unloading on any microplane, the response of the present model is path-independent. However, loading histories for which this siluation applics are nre (c.g., hydrostatic tension). For most loading histories, unloading happens at some microplanes, and then the re sponse depends on the path in the strain space. The great difference between the response curves in Fig. $7(d)$ or Fig. 8 is a result of strong path dependence. Modeling the path dependence of response, of course, is the main pupose of the present model.

As a demonstration of path dependence, we consider two histories leading to the same final stress state, one of them proportional and one nonproportional. Fig. 8(a) shows such histories for a combination of normal stresses $\sigma_{x}$ and $\sigma_{y}$, for which the principal stress direction does no rotate during loading, while Fig. 8(b) shows such histories for a combination of normal stress $\sigma_{x}$ and shear stress $\sigma_{x y}$, for which the principa stress direction rotates significantly. In the latter case, the final strain states differ more than in the former case. Apparently, rotation of the principal stress direction during loading amplifies the path dependence of response.

\section{Conclusions}

1. Strain softening due to tensile microcacking may be described with the microplane model in which the normal strain on a microplane of any orientation conforms to the same macroscopic strain tensor and the mormal stress on a microplane of any orientation is a function of only the normal strain on that same microplane. Using the virtual work principle, one can then obtain an explicit formula for the tangential stiffnesses, expressed as an integral over a unit hemisphere.

2. A desired correction of the elastic Poisson ratio may be achieved by an additional elastic strain.

3. Due to various possible combinations of loading and unloading on microplanes of various orientations, the model, in general, is path-dependent. Its main advantage is that it may be applied to loading path with rotating principal stress directions without violating any tensoria invariance restrictions of continuum mechanics. Progressive fracture and damage caused by such loadings is of considerable practical interest

4. Only scant test data are available at present for verification of the model. Those available are the direct tensile tests, which can be de scribed satisfactorily by the model. The data fitting is easy, since only two material parameters (aside from those for unloading) characterize the response.

5. For application in finite element programs with incremental loading, an efficient numerical evaluation of the tangential stiffness from an integral over the surface of a hemisphere is required. The integral over a unit hemisphere may be evaluated by numerical integration, which is tantamount to restricting the tensile microcracks or cracks to only a certain finite number of characteristic orientations, which correspond to integration points on the sphere. The numerically calculated uniaxial response curves converge with an increasing number of integration points even in the strain softening region.

6. Numerical integration formulas that are sufficiently accurate within the elastic (or hardening inelastic) range are not necessarily sufficiently accurate for the strain-softening range, and better formulas are needed. Some of the existing numerical integration formulas for the surface of a sphere (22) appear satisfactory, and some new, better formulas are developed. If errors of about $\pm 3 \%$ within the strain-softening range are acceptable, then one needs at least 21 integration points for general stress states and 14 points for plane stress or plane strain (or axisymmetric problems). If the errors within the strain-softening range should be kept below about $\pm 1 \%, 33$ integration points are needed, and if they should be kept below about $\pm 0.3 \%, 61$ points are needed for general stress states.

7. Various alternatives, such as a statically rather than kinematically constrained microplane system, and models in which the microplane system describes only the inelastic stress relaxations or the inelastic strains, have been explored, but appeared to be unstable for strain softening.

\section{ACKNOWLEDGMENT}

Partial support under Air Force Office of Scientific Research Grant No. 83-0009 to Northwestern University is gratefully acknowledged. Mary Hill is thanked for her excellent secretarial assistance.

\section{Appendix 1.-Direction Cosines for Formulas} BASED ON ICOSAHEDRON

The centers of the faces of an icosahedron correspond to the vertices of a dodecahedron. Denote the vertices of one pentagonal face of the dodecahedron as A, C, G, H and D. According to Fig. 1(e), $\overline{C D}=2 a \sin$ $54^{\circ}$, in which $a=$ length of the edge of the dodecahedron. The vertices $B, C$ and $D$, adjacent to vertex $A$; form an equilateral triangle whose height $\overline{\mathrm{EB}}[$ Fig. $1(f)]$ is $2 a \sin 54^{\circ} \sin 60^{\circ}$. Then $\overline{\mathrm{A}^{\prime} \mathrm{B}}=(4 / 3) a \sin 54^{\circ} \sin$ $60^{\circ}$, in which $A^{\prime}$ is the normal projection of vertex $A$ on the plane of triangle $B C D$. So $\sin \omega=(4 / 3) \sin 54^{\circ} \sin 60^{\circ}$, in which $\omega=\Varangle A^{\prime} A B$. The central angle $\beta=\Varangle A O B$ of adjacent vertices $A$ and $B$ then is $\beta=180^{\circ}$ $-2 \omega$. Evaluating $\beta$ we find that $\sin \beta=2 / 3$. (Similarly one can show that the cosine of the angle between two adjacent vertex directions of an icosahedron is $2 / 3$.)

Fixing the direction cosines for vertex 1 [Fig. 1(e)] as $\mathbf{n}_{1}=(1,0,0)$, we may choose $\mathbf{n}_{2}=(\cos \beta, 0, \sin \beta)$ for vertex 2 . Then $\mathbf{n}_{3}=\mathbf{R}_{1} \mathbf{n}_{2}, \mathbf{n}_{4}=$ $\mathbf{R}_{1}, \mathbf{n}_{3}, \mathbf{n}_{5}=\mathbf{R}_{2} \mathbf{n}_{1}, \mathbf{n}_{6}=\mathbf{R}_{2} \mathbf{n}_{5}, \mathbf{n}_{7}=\mathbf{R}_{3} \mathbf{a}_{1}, \mathbf{n}_{8}=\mathbf{R}_{3} \mathbf{a}_{7}, \mathbf{n}_{9}=\mathbf{R}_{\mathbf{4}} \mathbf{n}_{1}, \mathbf{n}_{10}$ $=\mathbf{R}_{1} \mathbf{a}_{9}$ in which $\mathbf{a}_{1}, \ldots \mathbf{a}_{10}=$ column matrices of direction cosines for vertices $1, \ldots 10$ [see the upper right node pattern in Fig. 2(a)]; and $\mathbf{R}_{1}$, $\mathbf{R}_{2}, \mathbf{R}_{3}$ and $\mathbf{R}_{4}=$ square $(3 \times 3)$ matrices for rotations by $120^{\circ}$ about vectors $n_{1}, n_{2}, n_{3}$ and $n_{4}$ (counterclockwise when looking against the vector directions). Furthermore, the centers of the faces of the dodecahedron correspond to vertices of a dual icosahedron; the unit direction 
vector for each center is obtained as the sum of the five direction vectors of all five vertices of one face of dodecahedron, divided by the length of the vector sum. The mid-edge unit direction vector is obtained as the sum of the two direction vectors of the vertices at the ends of the edge divided by the length vector sum. As a result, the 10 face-center directions of an icosahedron are $(1,0,0),(\cos \beta, 0, \sin \beta),[\cos \beta, \pm(\sqrt{3} / 2) \sin$ $\beta,-1 / 2 \sin \beta],\left[\cos ^{2} \beta-1 / 2 \sin ^{2} \beta, \pm(\sqrt{3} / 2) \sin \beta, 0.75 \sin 2 \beta\right]$ and $\left[\cos ^{2} \beta-0.5 \sin \beta, \pm(\sqrt{3} / 4) \sin \beta(3 \cos \beta \pm 1),-0.75 \sin \beta(\cos \beta \pm\right.$ $1]$, in which $\sin \beta=2 / 3$.

\section{APPEndix II.-RefERENCES}

1. Abramowitz, M., and Stegun, I. A., "Handbook of Mathematical Functions with Formulas, Graphs, and Mathematical Tables," Dover Publications, Inc., New York, N.Y., 1970, p. 894

2. Albrecht, I., and Collatz, L., "Zur numerischen Auswertung mehrdimensionaler Integrale," Zeitschrift für Angewandte Mathenatik und Mechanik, Band 38, Heft $1 / 2$, Jan./Feb., 1958, pp. 1-15

3. Batdorf, S. B., and Budiansky, B., "A Mathematical Theory of Plasticity Based on the Concept of Slip," NACA TN1871, Apr., 1949

4. Bažant, Z. P'., "Crack Band Model for Fracture of Geomaterials," Procerdings of the 4th International Conference an Numerical Methods in Gomechanics, $Z$. Eisenstein, ed. University of Alberta, Edmonton, Canada, Vol. 3, 1982, pp. 1137-1151.

5. Bažant, Z. Г., "Microplane Model for Strain-Controlled Inelastic Behavior," Mechanics of Enginecring Materials, Chapter 3, C. S. Desai and R. H. Gallagher, eds., John Wiley \& Sons, London, U.K., 1984, pp. 45-59.

6. Bażant, Z. P and Gambarova, P. "Crack Shear in Concrete: Crack Band Microplane Model" Journal of Struchural Engineering, ASCE, Vol. 110, No. 9, Sept., 1984, pp. 2015-2036.

7. Bažant, Z. P., and Oh, B. H., "Efficient Numerical Integration on the Surface of a Sphere." Report No. 83-2/428e. Center for Concrete and Geomaterials, Northwestern University, Evanston III., 1983; also Zcitschiff fïr Angetomedie Mathematik und Mechanik (ZAMM), Leipzig, Germany, 1985 (in press).

8. Bažant, Z P., and Oh, B. H., "Crack Band Theory for Fracture of Concrete." Materials and Structures (RILEM Paris), Vol 16,1983 pp. 155-177.

9. Bažant Z P and Oh B H "Microplane Model for Fracture Analysis of Concrete Structures," Procedings of the Symposium on the Interaction of NonNuclear Munitions with Structures, U.S. Air Force Academy, Colorado Springs, Colo., May, 1983, pp. 49-55.

10. Bažant, Z. P., and Oh, B. H. "Model of Weak Planes for Progressive Fracture of Concrete and Rock," Report No. 83-2/428m, Center for Concrete and Geomaterials, Northwestern University, Evanston, Ill. Feb. 1983.

11. Bažant, Z. P. Ozaydin, K., and Krizek, R. J. "Micromechanics Model for Creep of Anisotropic Clay," Journal of the Engincering Mechanics Ditision, ASCE, Vol. 101, 1975, pp. 57-78

12. Calladine, C. R.," "A Microstructural View of the Mechanical Properties of Saturated Clay," Geotechmique, Vol. 21, 1971, pp. 391-415.

13. Encyclopedic Dictionary of Mathematics, Vol. II, S. Lyanaga and Y. Kawada, eds. Massachusetts Institute of Technology Press, 1980, p. 1105.

14. Evans, R. H., and Marathe, M. S., "Microcracking and Stress-Strain Curves for Concrete in Tension." Matcrials and Structures (Paris), No. 1, Jan.-Feb., 1968, pp. 61-64.

15. Heilmann, H. G., Hilsdorf, H. H., and Finsterwalder, K., "Festigkeit und Verformung von Beton unter Zugspanungen," Deutscher Ausschuss für Stahlbeton, Heft 203, W. Ernst \& Sohn, West Berlin, Germany, 1969.

16. Hughes, B. P., and Chapman, G. P., "The Complete Stress-Strain Curve for
Concrete in Direct Tension," Bulletin RILEM (Paris) No. 30, 1966, pp. 95-97.

17. Pande, G. N., and Sharma, K. G. "Multi-Laminate Model of Clays-A Numerical Evaluation of the Influence of Rotation of the Principal Stress Axes." Report, Department of Civil Engineering, University College of Swansea, U.K. 1982; see also Procedings of the Sympositum on Implementalion of Computer Pro cedures and Stress-Strain Laws in Geotechnical Engineering, C. S. Desai and S K. Saxena, eds., held in Chicago, Ill., Aug., 1981, Acorn Press, Durham N.C., 1981, pp. 575-590

18. Pande, G. N., and Xiong, W., "An Improved Multi-Laminate Model of Jointed Rock Masses," Procecdings of the Internationnl Symposium on Numerical Modds in Gemedhanics, R. Dungar, G. N. Pande, and G A Studer, eds held in Zurich, Sept., 1982, Balkema, Rotterdam, 1982, pp. 218-226.

19. Reinhardt, H. W., and Cornelissen, H. A. W., "Post-Peak Cyclic Behavior of Concrete in Uniaxial Tensile and Alternating Tensile and Compressive Loading," Cement and Concrete Research, Vol. 14, 1984, pp. 263-270.

20. Rüsch, H., and Hilsdorf, H., "Deformation Characteristics of Concrete under Axial Tension," Voruntersuchungen, Bericht Nr. 44, Munich, Germany, May, 1963.

21. Sanders, J. L., "Plastic Stress-Strain Relations Based on Linear Loading Function," Procedings of the 2nd U.S. National Congress on Applied Mechanics, ASME 1955, pp. 455-460

22. Stroud, A. H., Approximate Calculation of Multiple Intcgrals," Prentice Hall Englewood Cliffs, N.I., 1971.

23. Taylor, G. I., "Plastic Strain in Metals," I. Inst. Metals, Vol. 62, 1938, pp $307-324$

24. Wittke, W., "New Design Concept for Underground Openings in Rocks," Finite Elements in Geomechanics, Chapter 13, G. Gudehus, ed., John Wiley. 1977 (see also Erzmetall, Vol. 26, No. 2, 1973, pp. 66-74)

25. Wood, D. N., "Exploration of Principal Stress Space with Kaolin in a Triaxial Apparatus," Geotechinique, Vol. 25, 1975, pp. 783-797.

26. Zienkiewicz, O. C., and Pande, G. N." "Time-Dependent Multi-Laminate Model of Rocks-A Numerical Study of Deformation and Failure of Rock Masses," Intcrnational Joumal of Numerical and Aualytical Methods in Geoucchanics, Vol. 1, 1977, pp. 219-247. 